\title{
Tools for studying populations and timeseries of neuroanatomy enabled through GPU acceleration in the Computational Anatomy Gateway
}

\author{
Daniel J. Tward \\ Center for Imaging Science \\ Department of Biomedical \\ Engineering \\ Johns Hopkins University \\ $3400 \mathrm{~N}$ Charles St \\ Baltimore, Maryland \\ dtward@cis.jhu.edu \\ Timothy Brown \\ Center for Imaging Science \\ Johns Hopkins University \\ $3400 \mathrm{~N}$ Charles St \\ Baltimore, Maryland \\ timothy@cis.jhu.edu \\ Chelsea S. Sicat \\ Center for Imaging Science \\ $3400 \mathrm{~N}$ Charles St \\ Baltimore, Maryland \\ chelsea@cis.jhu.edu
}

Anthony Kolasny

Center for Imaging Science

hns Hopkins University

3400 N Charles St

Baltimore, Maryland

anthony@cis.jhu.edu

\author{
Michael I. Miller \\ Center for Imaging Science \\ Department of Biomedical \\ Engineering \\ Co-Director of Kavli \\ Neuroscience Discovery \\ Institute \\ Johns Hopkins University \\ 3400 N Charles St \\ Baltimore, Maryland \\ mim@cis.jhu.edu
}

\begin{abstract}
The Computational Anatomy Gateway is a software as a service tool for medical imaging researchers to quantify changes in anatomical structures over time, and through the progression of disease. GPU acceleration on the Stampede cluster has enabled the development of new tools, combining advantages of grid based and particle based methods for describing fluid flows, and scaling up analysis from single scans to populations and timeseries. We describe algorithms for estimating average anatomies, and for quantifying atrophy rate over time. We report code performance on different sized datasets, revealing that the number vertices in a triangulated surface presents a bottleneck to our computation. We show results on an example dataset, quantifying atrophy in the entorhinal cortex, a medial temporal lobe brain region whose structure is sensitive changes in early Alzheimer's disease.
\end{abstract}

\section{CCS Concepts}

-Applied computing $\rightarrow$ Imaging;

Permission to make digital or hard copies of all or part of this work for personal or classroom use is granted without fee provided that copies are not made or distributed for profit or commercial advantage and that copies bear this notice and the full citation on the first page. Copyrights for components of this work owned by others than ACM must be honored. Abstracting with credit is permitted. To copy otherwise, or republish, to post on servers or to redistribute to lists, requires prior specific permission and/or a fee. Request permissions from permissions@acm.org.

XSEDE16, July 17-21, 2016, ,

(C) 2016 ACM. ISBN 978-1-4503-4755-6/16/07 . \$ $\$ 15.00$

DOI: http://dx.doi.org/10.1145/2949550.2949574

\section{Keywords}

science gateway, computational anatomy, medical imaging, neuroscience

\section{INTRODUCTION}

The Center for Imaging Science has been developing tools for quantitatively studying anatomical changes in neurodegenerative diseases such as Alzheimer's. These tools are offered to the medical imaging community through a software as a service model, hosted through the Computational Anatomy Gateway, a science gateway powered by the Stampede and Gordon clusters through XSEDE (www.mricloud. org) [5].

Computational anatomy quantifies structural changes in biology by constructing smooth mappings to identify corresponding points in atlas and target neurimaging data (see [8] for a recent overview). Properties of these mappings, such as local expansion or contraction, are used to describe and make inferences about biological processes. An example is shown in Fig. 1. Contours around a sagittal slice of the corpus callosum, a white matter tract connecting the hemispheres of the brain, are shown in cyan and red on the left. The cyan contour (our template), is mapped (blue contour) such that its shape closely matches the red contour (our target). The map itself can be seen through a deformed grid, and the local change in scale is used to quantify atrophy (tissue loss) of the target relative to the template.

In this work we show how these mapping techniques are used to estimate the average anatomical shape of a population, and to quantify atrophy by mapping onto a series of magnetic resonance imaging (MRI) scans of the same pa- 

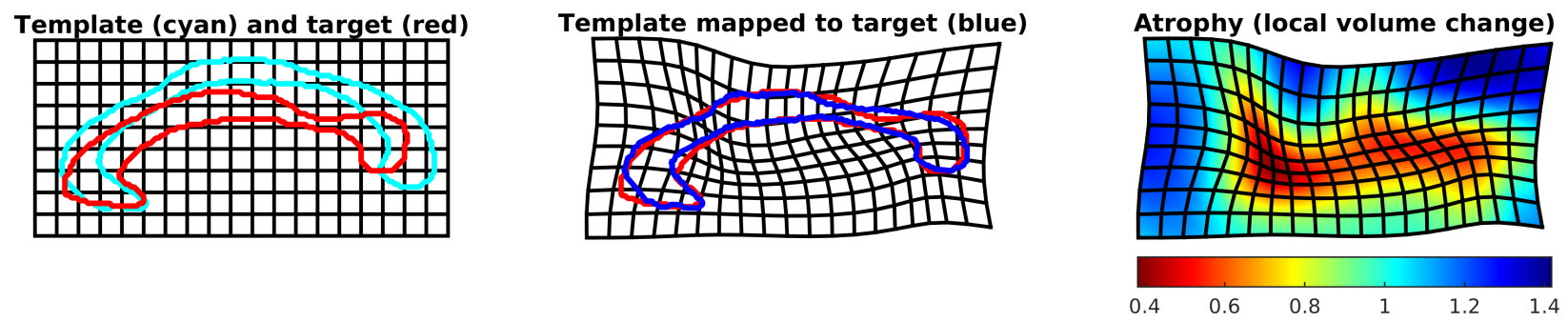

Figure 1: Left: example template and target contours describing a sagittal section of the corpus callosum. Middle: A diffeomorphism is used to deform the template to closely match the target (blue). Right: The diffeomorphism is used to measure areas of atrophy (red) and expansion (blue) to describe biological processes.

tients over time. These mapping procedures rely heavily on techniques from computational fluid dynamics, including both particle and grid based methods.

Traditionally, particle methods have been used when anatomy is described by labelled landmarks, or triangulated meshes (for example [12]). This allows the advantage of an efficient representation of anatomical form, and increased statistical power through fewer multiple comparisons. Grid based methods have been used when anatomy is described by a dense neuroimage (for example [1]). This has the advantage of removing unnecessary data processing, and better accounting for imaging noise. Parallel computing resources, in particular the NVIDIA Tesla K20 on Stampede, have allowed us to combine the advantages of these two methods [11], designing mapping tools that are both powerful and robust [10]. Additionally, larger memory has enabled us to move from studying single scans to populations and timeseries, more accurately accounting for variability.

Here we show how these tools are being used to study changes in the medial temporal lobe, a loci of the earliest changes in the onset of Alzheimer's disease (AD)[2].

\section{METHODS}

\subsection{Medial temporal lobe MRI}

T1 MRI neurimages from the publicly available Alzhimer's Disease Neuroimaging Initiative (ADNI) dataset were analyzed $^{1}$. One hundred patients were selected, matching the inclusion criteria for an upcoming drug trial. Subjects included were older adults (age $73 \pm 7$ years), 50\% male, education of $16 \pm 3$ years, with mild cognitive impairment. Each subject was scanned up to 5 times over 2 years. Medial temporal lobe structures were hand segmented, and the entorhinal cortex and immediately lateral cortex (the trans entorhinal region) were analyzed using our algorithms. For this study, a subset of 20 subjects were used.

For each subject, imaging data was rigidly aligned to the

\footnotetext{
${ }^{1}$ Data used in the preparation of this article were obtained from the Alzheimer's Disease Neuroimaging Initiative (ADNI) database ( adni.loni.usc.edu ). The ADNI was launched in 2003 as a public - private partnership, led by Principal Investigator Michael W. Weiner, MD. The primary goal of ADNI has been to test whether serial magnetic resonance imaging (MRI), positron emission tomography (PET), other biological markers, and clinical and neuropsychological assessment can be combined to measure the progression of mild cognitive impairment (MCI) and early Alzheimer's disease (AD). For up-to-date information, see www.adni-info.org.
}

baseline scan by minimizing a sum of square error cost function between pairs of T1 images. Between subjects, entorhinal cortex segmentations were aligned based on minimizing the distance between automatically placed landmarks.

\subsection{Equations of diffeomorphometry}

In computational anatomy, the background space of an image $\Omega \subset \mathbb{R}^{3}$ is deformed by a diffeomorphism $\varphi: \Omega \rightarrow \Omega$, which is generated by a flow under smooth time varying velocity vector fields $v$

$$
\varphi_{t}(x) \doteq x+\int_{0}^{t} v_{s}\left(\varphi_{s}(x)\right) d s
$$

The vector fields are modelled as belonging to a Hilbert space of smooth functions $V$ (see [14] for details), with norm given by $\|v\|_{V}^{2}=\langle v, v\rangle_{V}=\langle L v, L v\rangle_{L_{2}}=\int\left[L^{*} L v(x)\right]^{T} v(x) d x$ for $L$ a differential operator and $L^{*}$ its adjoint. This construction means smooth functions will have a small norm. Here $L$ will be chosen implicitly such that its Green's function $\left(K(x, y)\right.$ such that $\left(L^{*} L\right) K(\cdot, y)=\delta(\cdot-y)$ for $\delta$ the Dirac distribution) is a Gaussian of standard deviation $6 \mathrm{~mm}$ : $K(x, y)=\exp \left(-\frac{1}{2 \cdot 6^{2}}|x-y|^{2}\right)$.

This metric defines geodesics, or shortest length paths, on the group of diffeomorphisms, characterized by Euler's equation. By defining the momentum $\mu=L^{*} L v$, this geodesic equation can be written as [7]

$$
\dot{\mu}=-[D v]^{T} \mu
$$

where $D v$ denotes the $3 \times 3$ matrix of partial derivatives. The velocity vector field can be reconstructed by

$$
v(x)=\int K(x, y) \mu(y) d y .
$$

Working with geodesics reduces the complexity of our analysis: rather than needing to specify $v$ as a function of time, we only need to specify its initial condition.

Here we choose to model the momentum as a singular function, nonzero only on the boundary of the anatomical structures of interest. This representation is a parsimonious description of shape, whose dimension has been reduced from that of the background space $\left(\mathbb{R}^{3}\right)$ to that of a bounding surface $\left(\mathbb{R}^{2}\right)$. Under the assumption that neuroimages are a union of homogeneous tissue types, this representation does not lose any information [1].

We describe these surfaces parametrically through a function $f: U \subset \mathbb{R}^{2} \rightarrow \mathbb{R}^{3}$, and can specify the momentum and 


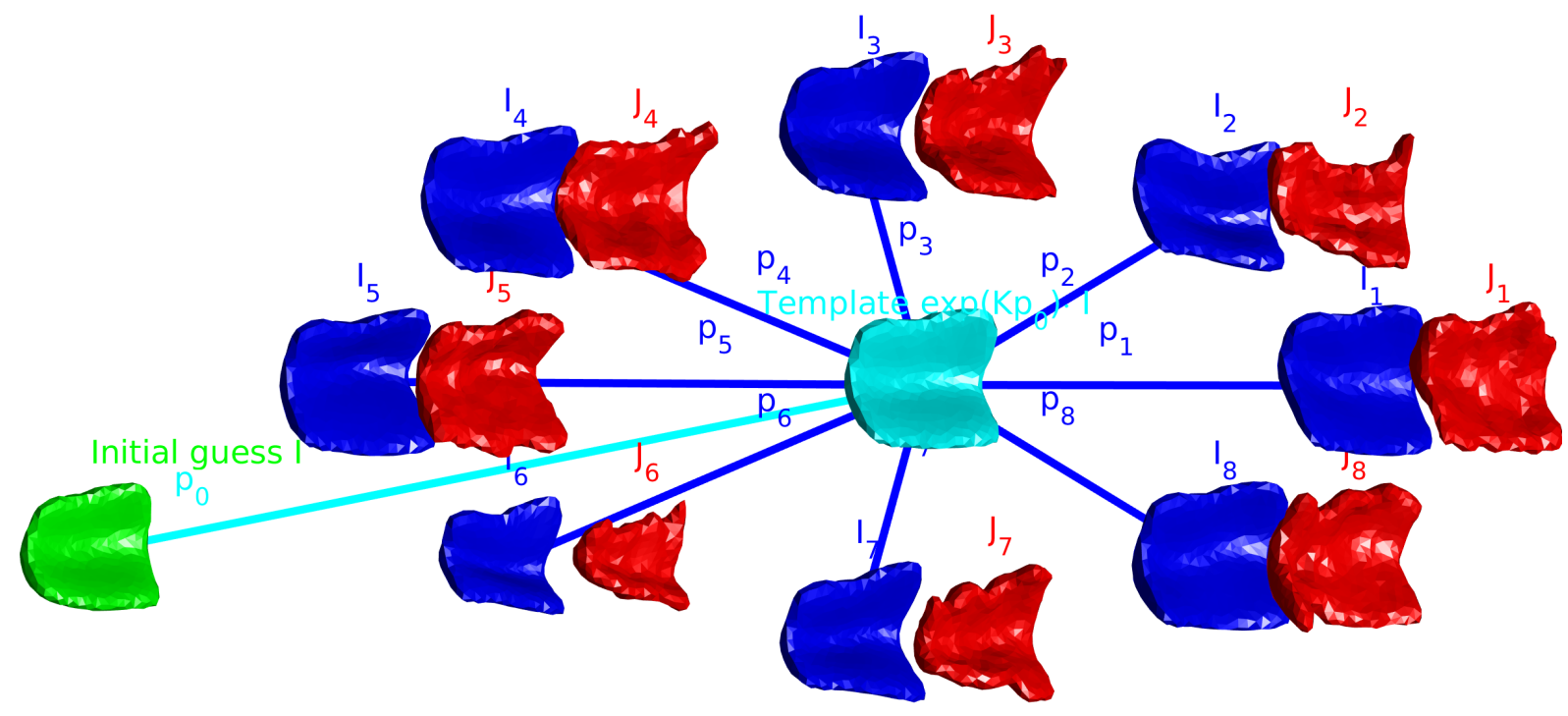

Figure 2: The template estimation setup is illustrated here using entorhinal cortex boundary surfaces. An initial guess (green) is transformed into template (cyan) which represents the average of the population. This template is mapped (blue) to closely match each subject (red), and its shape is determined by minimizing the cost function in $(7)$.

velocity through a function $p$ defined on the surface

$$
\begin{aligned}
\mu(\cdot) & \doteq \int_{U} \delta(\cdot-f(u)) p(u) d u \\
v(\cdot) & \doteq \int_{U} K(\cdot, f(u)) p(u) d u \\
\|p\|_{V^{*}}^{2} & \doteq\|v\|_{V}^{2}=\iint_{U \times U} p(u)^{T} K\left(f(u), f\left(u^{\prime}\right)\right) p\left(u^{\prime}\right) d u d u^{\prime}
\end{aligned}
$$

Surfaces are transformed by the diffeomorphism according to $f_{t}=\varphi_{t}\left(f_{0}\right)$. For notational convenience, we will write the solution to our system of geodesic equations by $\varphi_{t}=$ $\exp \left(t K p_{0}\right)$, where exp is the Riemannian exponential.

Here we represent these anatomical boundaries by triangulated surfaces. Our template surface has 676 vertices and 1348 faces. This is represented by a $676 \times 3$ double precision array. The function $p$ is represented using an array of the same size.

In this analysis we perform mappings between binary segmentation images that describe the entorhinal cortex. These are functions $I$ from $\Omega$ to $[0,1]$. They take the value 0 at locations outside a structure of interest, the value 1 inside it, and fractional values near the boundary depending on resolution and interpolation. An image is transformed by a diffeomorphism by acting on the right with the inverse: $I_{t} \doteq \varphi_{t} \cdot I_{0} \doteq I_{0} \circ \varphi_{t}^{-1}$.

Here images are represented by a regular double precision $3 \mathrm{D}$ array. The temporal lobe structures of interest are contained within a $42 \times 36 \times 45$ array at resolution $1 \mathrm{~mm} \times 1.2$ $\mathrm{mm} \times 1 \mathrm{~mm}$. Each of the $x, y, z$ components of the diffeomorphism $\varphi$, and velocity fields $v$ are represented using an array of the same size.

\subsection{Template estimation formulation}

We are given a family of $m$ neuroimages, each the baseline scan for a single subject, which we denote $J_{i}$, functions from the ambient space $\Omega \subset \mathbb{R}^{3}$ into $[0,1]$. We begin with a initial guess for our template: the surface $f$ is generated as an isocontour of the pointwise average of our population images, and the image $I$ is generated by filling the interior of this surface.

Following [6], we formulate a cost function as follows

$$
\begin{aligned}
& E\left(p_{0}, p_{1}, \ldots, p_{m}\right) \\
& =\frac{1}{2 \sigma_{p_{0}}^{2}}\left\|p_{0}\right\|_{V^{*}}^{2}+\sum_{i=1}^{m} \frac{1}{2 \sigma_{p_{i}}^{2}}\left\|p_{i}\right\|_{V^{*}}^{2}+\frac{1}{2 \sigma_{I_{i}}^{2}}\left\|I_{i}-J_{i}\right\|_{L_{2}}^{2}
\end{aligned}
$$

where we define $I_{i} \doteq \exp \left(K p_{i}\right) \cdot \exp \left(K p_{0}\right) \cdot I$. In this analysis each of the $\sigma$ weighting parameters is set to 1 .

We seek to determine the parameters $p_{0}, p_{1}, \ldots, p_{m}$ that minimize the cost. This will give us a template image $\exp \left(K p_{0}\right)$. $I$ and template surface $\exp \left(K p_{0}\right) \cdot f$ that characterize the average of our population in a minimum square distance sense.

This setup is illustrated in Fig. 2. The initial guess, $I$ or $f$ is shown at the bottom left in green. It is deformed using $p_{0}$ to the estimated template, shown in the center in cyan. This template is deformed under $p_{i}$ (blue) to match each member of the population (red). This illustration shows entorhinal cortex surfaces for the first 8 subjects in our dataset.

\subsection{Timeseries mapping formulation}

We are given a family of $n$ neuroimages for a single subject at times $t_{i}, i \in\{1, \ldots, n\}$, which we denote $J_{t_{i}}$ each being a function from the ambient space $\Omega \subset \mathbb{R}^{3}$ into $[0,1]$, and a template image $I: \Omega \rightarrow[0,1]$, and template surface $f: U \rightarrow \mathbb{R}^{3}$ estimated previously. We seek to match our template onto each image by estimating two geodesic 


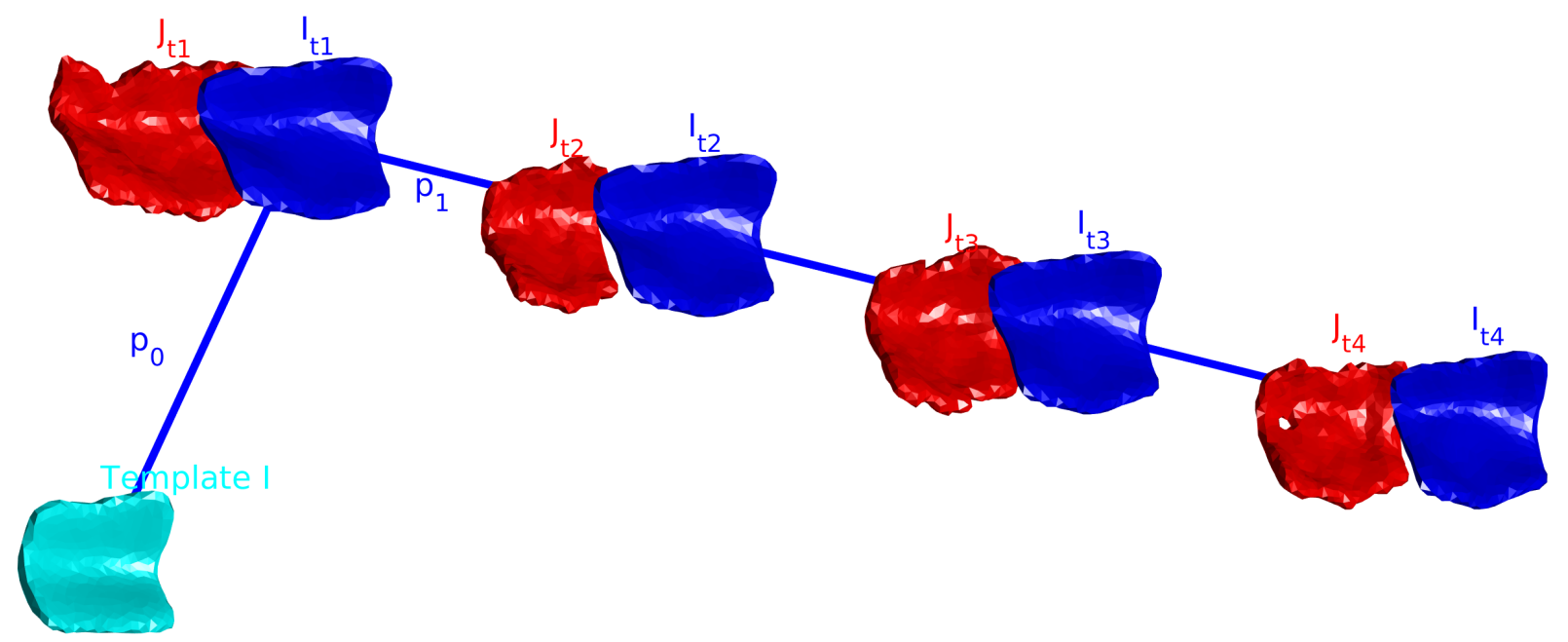

Figure 3: The timeseries mapping setup is illustrated here using entorhinal cortex boundary surfaces. A template (cyan) is transformed to the baseline scan (blue, top-left), and further transformed along a trajectory to closely match each segmentation in the timeseries (red). The trajectory is determined by minimizing (8).

trajectories. First from template to time $t_{1}$, then from $t_{1}$ through the timeseries to $t_{n}$.

We formulate a cost function as follows

$$
\begin{aligned}
& E\left(p_{0}, p_{1}\right) \\
& =\frac{1}{2 \sigma_{p_{0}}^{2}}\left\|p_{0}\right\|_{V^{*}}^{2}+\frac{1}{2 \sigma_{p_{1}}^{2}}\left\|p_{1}\right\|_{V^{*}}^{2}+\sum_{i=1}^{n_{t}} \frac{1}{2 \sigma_{I_{t_{i}}}^{2}}\left\|I_{t_{i}}-J_{t_{i}}\right\|_{L 2}^{2}
\end{aligned}
$$

where $I_{t_{i}}$ is the template image deformed to time $t_{i}$ via $\exp \left(K p_{1}\left(t_{i}-t_{1}\right)\right) \cdot \exp \left(K p_{0}\right) \cdot I$. In this analysis, each of the $\sigma$ weighting factor is set to 1 .

We seek to determine the parameters $p_{0}, p_{1}$ that minimize the cost. The deformation $\exp \left(K p_{0}\right)$ tells us how a given subject differs from the population average, and the deformation trajectory $\exp \left(K p_{1}\left(t-t_{1}\right)\right) \exp \left(K p_{0}\right)$ tells us how the subject's brain is changing over time. This procedure is essentially linear regression in a minimum square distance sense.

This setup is illustrated in Fig. 3. The template, $I$ or $f$ is shown at the bottom left in cyan. It is deformed using $p_{0}$ to the baseline scan, and then further deformed using $p_{1}$ to closely match each subsequent scan in the timeseries. Notice in this example the variable anterior-posterior (left-right) extent of the entorhinal cortex segmentation (e.g. the second timepoint appears much smaller). These inconsistencies in anatomical definitions are filtered out by mapping onto the whole timeseries simultaneously. Mapping onto each individual scan result in much more variability.

\subsection{Adjoint algorithm}

In each case, the minimization is undertaken using gradient descent, where the gradient is calculated using an adjoint algorithm. That is, the gradient with respect to each image matching term is calculated as

$$
\frac{1}{\sigma_{I_{i}}^{2}}\left(I_{i}-J_{i}\right)
$$

and transported back to the appropriate template, following a linearized verson of the dynamics. Summing over all the error terms gives the cost function gradient.

We omit the details here and refer the reader to [13] or [4]. The computation involves only variations of the interpolation and weighted sums described below.

\subsection{Computation}

Each mapping algorithm was implemented on the GPU using OpenCL.

Surfaces are deformed by first computing the value of the velocity at each vertex

$$
\dot{f}_{t}\left(u_{l}\right)=\sum_{m} K\left(f_{t}\left(u_{l}\right), f_{t}\left(u_{m}\right)\right) p_{t}\left(u_{m}\right)
$$

and then flowing forwards according to Euler's method

$$
f_{t+1}\left(u_{l}\right)=f_{t}\left(u_{l}\right)+\Delta t \dot{f}_{t}\left(u_{l}\right)
$$

These calculations are parallelized on the GPU over each output variable.

Dense velocity fields are generated by

$$
v_{t}\left(x_{i j k}\right)=\sum_{l} K\left(x_{i j k}, f_{t}\left(u_{l}\right)\right) p_{t}\left(u_{l}\right)
$$

with $x_{i j k}$ being the $i, j, k$-th gridpoint in our 3D array, and $u_{l}$ the $l$-th vertex in our triangulated surface. Note that this operation is "like" a convolution, but because $f_{t}\left(u_{l}\right)$ is not regularly spaced, fast Fourier transform techniques cannot be used. These sums are parellelized on the GPU with one thread per output $i j k$.

Diffeomorphisms are generated from $v$ using a technique from numerical weather prediction called semi-Lagrangian 
advection [9], with

$$
\varphi_{t+1}^{-1}\left(x_{i j k}\right)=\varphi_{t}^{-1}\left(x_{i j k}-\Delta t v_{t}\left(x_{i j k}\right)\right),
$$

and the evaluation is performed through trilinear interpolation.

Images are also deformed through trilinear interpolation

$$
I_{t}\left(x_{i j k}\right)=I_{0}\left(\varphi_{t}^{-1}\left(x_{i j k}\right)\right)
$$

Both these interpolation operations are parallelized with one thread per output $i j k$.

In general, each calculation needed can be expressed as an interpolation, or as a weighted sum over vertices.

Tests were performed on a Stampede GPU node with 2 8-core Intel Xeon CPUs (E5-2680) at $2.7 \mathrm{GHz}$, though the software examined did not parallelize over multiple CPU cores. GPU computations were performed on a Tesla K20m card in double precision arithmetic, with a maximum work group size of 1024 and 13 parallel compute cores. Global work group size was set to the nearest integer multiple of CL_KERNEL_PREFERRED_WORK_GROUP_SIZE_MULTIPLE times CL_DEVICE_MAX_COMPUTE_UNITS (i.e. 13 on this card) larger than the desired number of output variables, and local work group size was chosen automatically by OpenCL through calling the enqueueNDRangeKernel function with the cl: : NullRange argument.

\subsection{Markers of shape change}

At each vertex in our template triangulated surface, we measure the determinant of the $3 \times 3$ Jacobian of the diffeomorphism calculated for each subject at each timepoint. This measure tells us by what factor tissue is growing or shrinking in a small region around this vertex. The value 1 represents no change, values less than 1 represent atrophy or tissue loss, and values greater than 1 represent growth or tissue gain.

We fit the determinant of Jacobian to a linear model, and estimate a mean and atrophy rate through least squares. This gives atrophy rate as a biomarker for $\mathrm{AD}$ progression, at each vertex of our triangulated surface template.

\section{RESULTS}

\subsection{Computational performance}

At XSEDE2015 [11] we presented early results showing a dramatic increase in computation speed on the GPU versus $\mathrm{CPU}$ for the combined particle and grid methods. We have since developed these specific algorithms, and present results that illustrate how they perform on the Stampede cluster for differently sized datasets.

For the following results, total wall clock time was measured by comparing timestamps on empty files created before and after code execution, and computation time per iteration was determined by dividing this elapsed time by 100 iterations of gradient descent optimization.

\subsubsection{Number of surface vertices}

For this experiment vertex points were placed in a uniformly spaced 3D grid spanning the image volume, rather than on a surface contouring an anatomical structure. Shown in Fig. 4 are the timing results of our timeseries mapping procedure, with only a single timepoint, while varying the number of vertices in our template. Above approximately

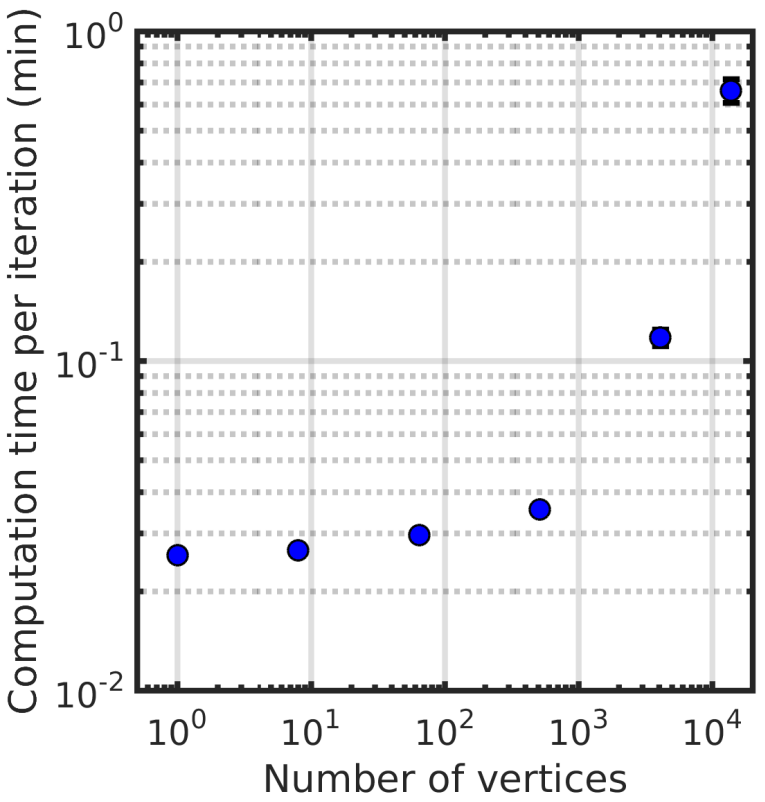

Figure 4: For our fixed image size, the computation time per iteration of optimization is shown as a function of an increasing number of surface vertices.

1000, the time begins increasing quickly. This motivates our use of a template with slightly less than 1000 vertices.

\subsubsection{Number of subjects in template estimation}

For this experiment a template shape was estimated using differently sized subsets of baseline scans from our ADNI population. Shown in Fig. 5 are the timing results of our template estimation procedure, varying the number of subject images. It can be seen that computation time increases roughly linearly with number of patients in our population, indicating that there are no bottlenecks with regard to number of subjects at this scale.

\subsubsection{Number of scans in timeseries mapping}

For this experiment we used one ADNI subject with images at five timepoints, and performed mapping on subsets of the first 1 to 5 scans. Shown in Fig. 6 are the timing results of our timeseries mapping procedure, varying the number of subject images. It can be seen that computation time increases roughly linearly with number of scans, indicating that there are no bottlenecks with respect to number of timepoints at this scale.

\subsection{Atrophy rate}

The results of our template estimation and timeseries mapping procedure are shown in Fig. 7. One sees atrophy throughout the cortex, but particularly near the most inferior point and lateral to it, an area known as the sulcal region of the entorhinal cortex [3]. This pattern is consistent with [2], where these changes could only be identified in histology of post mortem brains.

\section{CONCLUSIONS}

GPU nodes on the Stampede cluster have allowed us to 


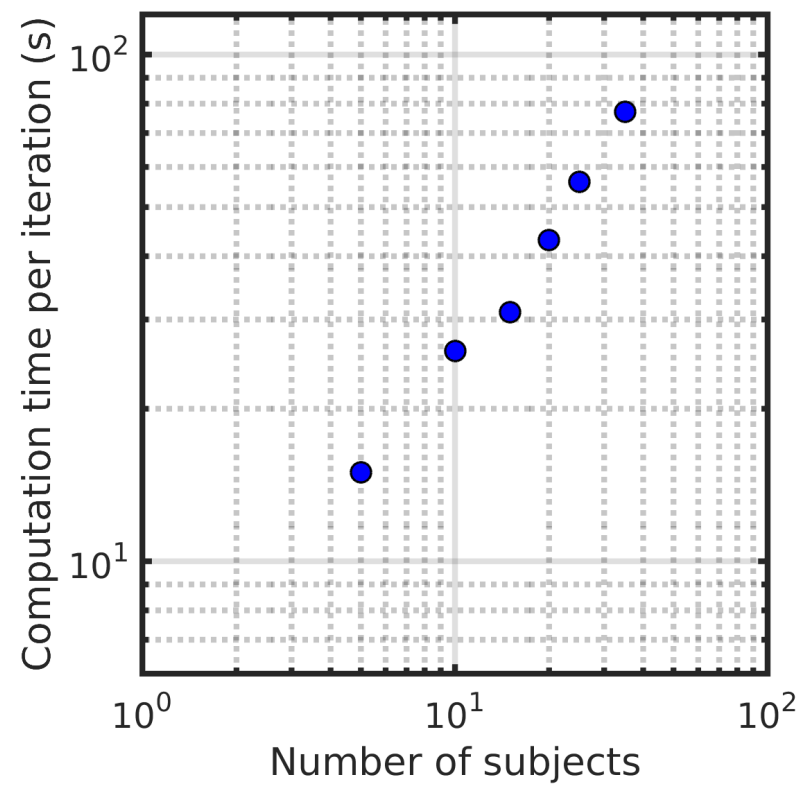

Figure 5: For our fixed image size, and fixed template vertices, we show the computation time for one iteration of optimization in template estimation while varying the number of patients.

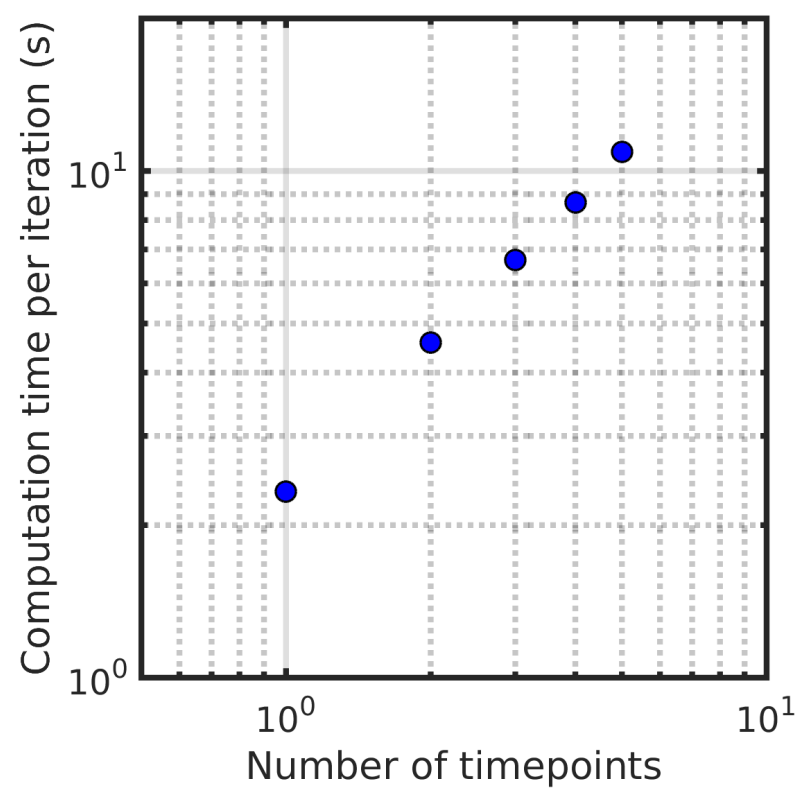

Figure 6: For our fixed image size, and fixed template vertices, we show the computation time for one iteration of optimization in timeseries mapping while varying the number of scans.

explore a class of computational anatomy algorithms that combine the advantages of particle based and grid based methods. At XSEDE2015 [11] we demonstrated that mapping a single template to a single target in this framework

\section{Atrophy rate (\%/year)}
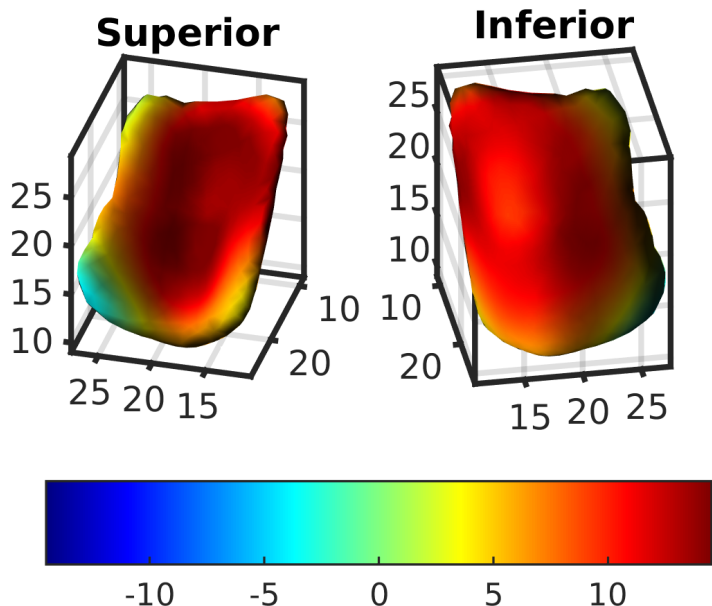

Figure 7: The estimated template surface, contouring the entorhinal and surrounding cortex, is shown from a superior view on the left, and an inferior view on the right. The far left and far right show the medial side, or entorhinal cortex, while the middle shows the lateral side, or trans-entorhinal region. Each vertex is colored by the atrophy rate estimated from our linear model.

could be infeasible on a CPU for some typically sized images and surfaces. This year, through GPU technology, we are able to not only perform these basic mappings, but expand the size of datasets analyzed and complexity of algorithms used. Here we have characterized the performance of two algorithms, template estimation and timeseries mapping. We have identified the number of vertices in our triangulated surface template as an important computational bottleneck, and will investigate ways to improve performance.

We have applied these methods to studying a population of subjects in the early stages of Alzheimer's disease, and have identified regions of atrophy that are consistent with post-mortem techniques.

Since code was developed in OpenCL, it can be compiled on different parallel architectures. Future work will include investigating the performance of our algorithms using the Intel Xeon Phi, which is available on 6400 nodes on Stampede, in addition to the NVIDIA Tesla K20 GPU which has only 128 available nodes.

\section{ACKNOWLEDGEMENTS}

This project was supported by the National Center for Research Resources and the National Institute of Biomedical Imaging and Bioengineering of the National Institutes of Health through Grant Number P41 EB015909.

This work was supported by the Kavli Foundation .

This work used the Extreme Science and Engineering Discovery Environment (XSEDE), which is supported by National Science Foundation grant number ACI-1053575. 
Data collection and sharing for this project was funded by the Alzheimer's Disease Neuroimaging Initiative (ADNI) (National Institutes of Health Grant U01 AG024904) and DOD ADNI (Department of Defense award number W81XWH12-2-0012). ADNI is funded by the National Institute on Aging, the National Institute of Biomedical Imaging and Bioengineering, and through generous contributions from the following: AbbVie, Alzheimer's Association; Alzheimer's Drug Discovery Foundation; Araclon Biotech; BioClinica, Inc.; Biogen; Bristol-Myers Squibb Company; CereSpir, Inc.; Eisai Inc.; Elan Pharmaceuticals, Inc.; Eli Lilly and Company; EuroImmun; F. Hoffmann-La Roche Ltd and its affiliated company Genentech, Inc.; Fujirebio; GE Healthcare; IXICO Ltd.; Janssen Alzheimer Immunotherapy Research \& Development, LLC.; Johnson \& Johnson Pharmaceutical Research \& Development LLC.; Lumosity ; Lundbeck; Merck \& Co., Inc.; Meso Scale Diagnostics, LLC.; NeuroRx Research; Neurotrack Technologies; Novartis Pharmaceuticals Corporation; Pfizer Inc.; Piramal Imaging; Servier ; Takeda Pharmaceutical Company ; and Transition Therapeutics . The Canadian Institutes of Health Research is providing funds to support ADNI clinical sites in Canada. Private sector contributions are facilitated by the Foundation for the National Institutes of Health ( www.fnih.org ). The grantee organization is the Northern California Institute for Research and Education, and the study is coordinated by the Alzheimer's Disease Cooperative Study at the University of California, San Diego. ADNI data are disseminated by the Laboratory for Neuro Imaging at the University of Southern California .

\section{REFERENCES}

[1] M. F. Beg, M. I. Miller, A. Trouvé, and L. Younes. Computing large deformation metric mappings via geodesic flows of diffeomorphisms. International journal of computer vision, 61(2):139-157, 2005.

[2] H. Braak and E. Braak. Neuropathological stageing of alzheimer-related changes. Acta Neuropathologica, 82:239-259, 1991. http://dx.doi.org/10.1007/BF00308809.

[3] S.-L. Ding and G. W. Van Hoesen. Borders, extent, and topography of human perirhinal cortex as revealed using multiple modern neuroanatomical and pathological markers. Human brain mapping, 31(9):1359-1379, 2010.

[4] S. Durrleman, S. Allassonnière, and S. Joshi. Sparse adaptive parameterization of variability in image ensembles. Int. J. Comput. Vision, 101(1):161-183, Jan. 2013.

[5] S. Jain, D. J. Tward, D. S. Lee, A. Kolasny, T. Brown, J. T. Ratnanather, M. I. Miller, and L. Younes. Computational anatomy gateway: Leveraging xsede computational resources for shape analysis. In Proceedings of the 2014 Annual Conference on Extreme Science and Engineering Discovery Environment, XSEDE '14, pages 54:1-54:6, New York, NY, USA, 2014. ACM.

[6] J. Ma, M. I. Miller, and L. Younes. A bayesian generative model for surface template estimation. International Journal of Biomedical Imaging, 2010, 2010.

[7] M. I. Miller, A. Trouvé, and L. Younes. Geodesic shooting for computational anatomy. Journal of mathematical imaging and vision, 24(2):209-228, 2006.

[8] M. I. Miller, L. Younes, and A. Trouve. Diffeomorphometry and geodesic positioning systems for human anatomy. Technology (Singapore World Science), 2:36, 2014. http://dx.doi.org/10.1142/S2339547814500010.

[9] A. Staniforth and J. Côté. Semi-lagrangian integration schemes for atmospheric models-a review. Mon. Wea. Rev., 119(9):2206-2223, sep 1991.

[10] D. Tward, J. Jovicich, A. Soricelli, G. Frisoni, A. Trouve, L. Younes, and M. Miller. Machine learning in medical imaging: 5th international workshop, mlmi 2014, held in conjunction with miccai 2014, boston, ma, usa, september 14, 2014, proceedings. volume 8679. Springer, 2014.

[11] D. J. Tward, A. Kolasny, N. Charon, M. I. Miller, and L. Younes. Gpu acceleration on the stampede cluster for the computational anatomy gateway. XSEDE '15, 2015. https://conferences.xsede.org/-/

gpu-acceleration-on-the-stampede-cluster-for-the-computational-ana

[12] M. Vaillant and J. Glaunes. Surface matching with currents. Information Processing in Medical Imaging, 19:381-392.

[13] F.-X. Vialard, L. Risser, D. Rueckert, and C. Cotter. $3 \mathrm{~d}$ image registration via geodesic shooting using and efficient adjoint calculation. Journal International Journal of Computer Vision, 97(2):229-241, April 2012.

[14] L. Younes. Shapes and Diffeomorphisms, volume 171. Series: Applied Mathematical Sciences, 2010. 